\title{
Le cadre conceptuel de la législation sur les retraites durant la première moitié $\mathrm{du} \mathrm{XX}^{\mathrm{e}}$ siècle
}

\section{Anna Aleksandrova}

\section{(2) OpenEdition}

1 Journals

\section{Édition électronique}

URL : https://journals.openedition.org/rdctss/1209

DOI : 10.4000/rdctss. 1209

ISSN : 2262-9815

Éditeur

Centre de droit comparé du travail et de la sécurité sociale

\section{Édition imprimée}

Date de publication : 1 avril 2020

Pagination : 102-113

ISSN : 2117-4350

\section{Référence électronique}

Anna Aleksandrova, "Le cadre conceptuel de la législation sur les retraites durant la première moitié du XXe siècle », Revue de droit comparé du travail et de la sécurité sociale [En ligne], 1 | 2020, mis en ligne le 01 novembre 2021, consulté le 11 novembre 2021. URL : http://journals.openedition.org/rdctss/ 1209 ; DOI : https://doi.org/10.4000/rdctss. 1209

\section{cc)}

Revue de droit comparé du travail et de la sécurité sociale est mise à disposition selon les termes de la Licence Creative Commons Attribution - Pas d'Utilisation Commerciale - Pas de Modification 4.0 International. 


\section{LE CADRE CONCEPTUEL DE LA LÉGISLATION SUR LES RETRAITES DURANT LA PREMIÈRE MOITIÉ DU XX' SIÈCLE*}

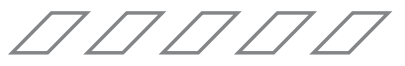

\section{RÉSUMÉ}

Cette contribution analyse les différents concepts politiques et juridiques à l'origine de la législation sur les retraites - les théories du droit naturel, du contrat social, de la solidarité, de l'égalitarisme, etc. - incarnés dans les lois sur les retraites adoptées au Royaume-Uni, en France et en Russie dans la première moitié du $X X^{e}$ siècle.

MOTS CLÉS : Retraite, concept, solidarité, assurance, assistance.

\section{ABSTRACT}

This study analyzes the different political and legal concepts behind pension legislation theories of natural law, the social contract, solidarity, egalitarianism, etc. - embodied in the pension laws adopted in United Kingdom, France and Russia in the first half of the 20th century.

KEYWORDS : Pension, Concept, Solidarity, Insurance, Assistance.

* Cette étude a été financée par RFBR, numéro de projet 20-011-00252. 
es recherches concernant les fondements conceptuels de la législation sur les retraites sont déterminées par un certain nombre de raisons, notamment:

- la crise des systèmes modernes d'assurance retraite (les nombreuses tentatives de réforme de ces systèmes - plus ou moins réussies - dans différents pays en témoignent);

- la crise générale de "l'État providence » (Welfare State) qui s'est manifestée à la fin du $X X^{e}$ siècle $^{1}$, provoquée par des facteurs objectifs et subjectifs comme la diminution du taux de croissance économique dans les pays développés, la détérioration de la situation démographique, la modification des relations de travail et des formes d'emploi, l'affaiblissement des principes de solidarité et la progression de l'individualisme, etc.) ;

- les tentatives visant à développer un modèle alternatif à l'État social (Workfare State $^{2}$, État animateur ${ }^{3}$, etc.).

En analysant les idées, concepts et principes théoriques qui se trouvent à l'origine de la législation sur les retraites dans certains pays européens (en l'occurence la France, le Royaume-Uni et la Russie), la présente étude cherche à expliquer le vecteur du développement de la législation sur les retraites (I) et à déterminer ses perspectives d'avenir (II). La première moitié du XXe siècle est ici choisie comme période de référence, car c'est à cette époque que les premières lois sur les pensions ont été adoptées et que des régimes nationaux de retraite ont été créés dans les pays susmentionnés. Plus tard, dans la seconde moitié du XXe siècle, ces régimes ont achevé leur développement et, à la fin du XXe siècle, ils se sont plus ou moins trouvés en crise.

\section{I - GENĖSE DU CADRE CONCEPTUEL DE LA LÉGISLATION SUR LES RETRAITES}

Les fondements théoriques de la législation sur les retraites ont leurs origines dans des pratiques sociales bien antérieures au XXe siècle. Les formes traditionnelles de soutien aux personnes âgées résidaient alors dans des formes de solidarités familiales et corporatives, ainsi que dans les œuvres caritatives (religieuse, privée

1 P. Rosanvallon, La Nouvelle Question sociale. Repenser l'Etat-providence, Seuil, 1995.

2 V.E. Chirkin, "Les valeurs universelles et l'État moderne », in L'État et le droit, 2002, n² 2, p. 12.

3 G. Vallée. «Diversité des modes d'action de l'État dans le domaine social et le système de relations industrielles », in L'État à l'épreuve du social, Paris, 1998, p. 257. 
ou publique). Ces formes de soutien sont à l'origine des principaux éléments constitutifs du système moderne des retraites, à savoir l'assistance et l'assurance. Notons que, du point de vue de la science économique, ces éléments s'apparentent aux différentes méthodes de gestion du risque social ${ }^{4}$.

L'assurance retraite est basée sur le principe de solidarité. Elle provient du concept de charité, c'est-à-dire du soutien gratuit, et implique le partage des risques dans le sens où elle prévoit une compensation des risques proportionnelle à la participation de chacun au financement des fonds de protection sociale.

L'assistance retraite, au contraire, relève d'une autre méthode de gestion du risque social : l'absorption. II s'agit d'une compensation financière directe des conséquences des risques sociaux, en fonction de leur gravité et du montant des garanties sociales établies.

Les principes fondateurs de l'assistance comme de l'assurance en matière de retraite, remontent à l'ère du Monde Antique et du Moyen Âge. Dès l'Antiquité, apparaissent les prémices des politiques sociales : aider les affamés en période de mauvaise récolte, faire l'aumône, etc. L'État offrait des mesures de soutien aux soldats handicapés, aux veuves et aux orphelins. Dans les anciennes Lois de Manu en Inde, la mendicité était reconnue comme une source de subsistance (article 116 du chapitre $X)^{5}$. Dans la Rome Antique (ler siècle avant JC), il existait un collège de mutualité pour les citoyens libres de Rome, qui collectait des cotisations et allouait le soutien aux personnes âgées, malades et handicapés ${ }^{6}$.

Au Moyen Âge, l'Église chrétienne a commencé à jouer un rôle important en matière d'aide caritative et d'organisation de la charité publique.

Les principes de charité, décrits par les philosophes chrétiens, consistaient notamment dans :

- la nature volontaire, désintéressée et non officielle des dons (bienfaits);

- l'attitude sélective des bienfaiteurs à l'égard de ceux qui en ont besoin;

- l'irréprochabilité morale des intentions du bienfaiteur?

$\mathrm{Au} \mathrm{XVle} \mathrm{siècle} \mathrm{en} \mathrm{Europe,} \mathrm{un} \mathrm{système} \mathrm{d'assistance} \mathrm{publique} \mathrm{commence} \mathrm{à} \mathrm{se}$ former pour aider les personnes misérables et handicapées et sanctionner au contraire les mendiants physiquement aptes au travail. Par exemple en Angleterre, la loi des pauvres (poor law) adoptée en 1601 prévoyait la création de « maisons du travail » (workhouses) pour les voleurs, vagabonds, mendiants ou indigents en capacité de travailler. En échange d'un abri et d'une maigre nourriture, ces derniers étaient mis à contribution, contraints au travail et soumis à des peines corporelles.

Le prototype originel de l'assurance retraite réside dans la mutualité des corporations médievales. Les mécanismes collectifs de soutien mutuel, au sein de corporations professionnelles, de communautés rurales ou urbaines, avaient en

4 E.V. Ageeva, Systèmes de retraite modernes: manuel de formation, Irkoutsk, 2015, p. 11.

5 Lois de Manu, Moscou, 1960, p. 225.

6 Lecture sur l'histoire de la Rome antique, éd. S.L. Utchenko, Moscou, 1962, p. 508.

7 O. A. Chernega, Modèle juridique de la charité et des organisations caritatives : aspects civils et sociologiques, Moscou, 1998, p. 10. 
effet pour fonction de "compenser " l'insuffisace des politiques sociales menées par l'État féodal.

Le soutien aux personnes nécessiteuses était davantage régi par les us et coutumes de l'époque, ou par des normes corporatives, morales et religieuses, tant le règlement législatif en la matière était déclaratif et fragmentaire ${ }^{8}$. Ainsi, à l'ère du Monde Antique et du Moyen-Âge, les mesures de soutien aux personnes les plus défavorisées restaient rares et individualisées, sans système public unique d'assistance.

II fallut attendre que les philosophes et juristes des $X V \|^{e}$ et $X X^{e}$ siècles s'intéressent et étudient le droit à l'assistance et à la sécurité sociale pour que le droit individuel à la retraite puisse être enfin énoncé et libellé. Les idées des Lumières ont joué un rôle important dans le processus de consécration législative. De nouvelles approches philosophiques ont ainsi permis de mieux comprendre l'interaction entre l'individu et l'État, le développement et la justification du concept des droits humains naturels, y compris la vie, la liberté et la dignité de l'individu.

En particulier, Thomas Hobbes a souligné l'égalité fondamentale de toutes les personnes, écrivant que les lois naturelles sont « la justice, l'impartialité, la modestie, la charité » et que "tous les individus sont également libres devant la nature " son ouvrage Deux traités sur le gouvernement, John Locke avançait quant à lui la thèse du "droit à la défense " $^{10}$. Enfin, Charles Montesquieu affirmait pour sa part que l'État était obligé de fournir aux citoyens de quoi s'abriter, se nourrir et se vêtir ${ }^{11}$. Ces philosophes sont aujourd'hui considérés comme les fondateurs de la théorie du contrat social qui, partant du fait que les relations sociales sont artificielles, suggère un élément de consentement volontaire ${ }^{12}$. Ainsi, selon Pierre Rosanvallon, "le concept d'assurance devient une sorte de substitut à un contrat social, car il suppose des principes similaires de concentration des biens et de protection sociale ${ }^{13}$.

Les mécanismes de soutien mutuel (y compris l'assurance) sont apparus bien avant le XVIII e siècle mais n'étaient utilisés que pour l'assurance de la propriété. G.W. Leibniz fut le premier philosophe à considérer l'assurance sociale comme un instrument de justice sociale, estimant que le principe de mutualité obligatoire devait protéger les personnes contre les risques sociaux ${ }^{14}$.

N. de Condorcet considérait pour sa part l'assurance sociale comme un moyen de lutter contre les inégalités et la pauvreté. Dans son ouvrage Esquisse d'un tableau historique des progrès de l'esprit humain ${ }^{15}$, Condorcet souligne ainsi la possibilité

8 N.V. Putilo, Fondements de la réglementation juridique des droits sociaux,Moscou, 1999, p. 13.

9 T. Hobbes et E. Canetti, Leviathan. Comment nait un monstre de pouvoir, Moscou, 2019, p. 11.

10 J. Locke, Two Treatises of Government, Cambridge, 1988, p. 285.

11 Ch. Montesquieu, De l'esprit des lois, Londres, 1786, vol. 3, p. 119.

12 P. Rosanvallon, op. cit., p. 19.

13 ld.

14 G. Grua, La Justice humaine selon Leibniz, Paris, PUF, 1956, p. 336.

15 N. de Condorcet, Esquisse d'un tableau historique des progrès de l'esprit humain, Moscou, 2010, p. 230. 
d'utiliser des mécanismes d'assurance fondés sur le principe de solidarité pour la protection sociale des personnes âgées, ajoutant par ailleurs que les institutions d'assurance sociale, publiques ou privées (résultant d'une « initiative privée») sont "I'une des plus grandes bonnes actions $»^{16}$.

La doctrine de l'assurance vie des salariés, fondée par Etienne Clavier, est présentée dans son ouvrage «Prospectus de l'établissement des assurances sur la vie "(1788) ${ }^{17}$. Clavier pensait que l'utilisation de mécanismes d'assurance permettrait une approche complètement nouvelle du problème de la pauvreté, éliminant l'instabilité de la vie humaine qui crée le malheur.

Cependant, les idées d'assurance-retraite formulées au XVIII siècle n'ont pas trouvéun largesoutien danslescercles publicsetpolitiques. Denombreuxphilosophes et économistes pensaient qu'une telle approche, destinée à résoudre les problèmes sociaux, pouvait engendrer le "parasitisme » des citoyens. Expliquant ce phénomène, Pierre Rosanvallon écrit : "Pendant longtemps, la prévoyance obligeant une personne à prendre soin de son avenir s'était opposée à l'assurance qui pourrait favoriser la négligence. Ce n'est qu'à la fin du XIXe siècle que le mécanisme de l'assurance individuelle a été reconnu comme une institution adéquate et moralement acceptable pour résoudre les problèmes sociaux ${ }^{18}$.

On pourrait citer l'avis de John Stuart Mill qui écrit, au milieu du XIXe siècle, que "le droit au soutien généré par la pauvreté est l'un des droits les plus incontestables qui puisse exister ${ }^{19}$. Parallèlement, il pensait que " l'aide est un tonique et non un sédatif : elle stimule plutôt qu'elle ne noie la capacité d'une personne à agir, la condition indispensable est que cette aide ne remplace pas le besoin d'auto-assistance, c'est-à-dire ne remplace pas son propre travail, ses compétences et sa prudence ${ }^{20}$. Dans son ouvrage Principles of Political Economy with some of their applications to social philosophy, J. S. Mill préconise ainsi les lois anglaises contre la pauvreté.

Jusqu'à la fin du XIXe siècle, c'est le courant de pensée du "laissez-faire »c'est-à-dire le non-interventionniste - qui a prévalu dans les politiques de l'État vis-à-vis de la société. Le principal adage de ce courant était « le recours à la force du travailleur, à sa prévoyance et à son épargne $»^{21}$.

Cependant, l'étroitesse de cette approche devenait de plus en plus évidente dans la mesure où un grand nombre de travailleurs percevant de bas salaires et ne pouvant donc pas épargner d'argent pour leur vieillesse, les appels à la prudence et à la prévoyance restaient de ce fait une expression vide de sens ${ }^{22}$. Inversement, les travailleurs réunis dans le cadre d'une production à grande échelle se sentaient

16 Ibid., p. 231

17 E. Clavière, «Prospectus de l'établissement des assurances sur la vie », Risques, Paris, 1990, $n^{\circ} 1$, p. 134.

18 P. Rosanvallon, op. cit., p. 21.

19 J. S. Mill, Fondements de l'économie politique avec quelques applications à la philosophie sociale, Moscou, 2007, p. 973.

20 Ibid., p. 974.

21 A. I. Ogus et E. M. Barendt, The Law of Social Security, London, 1978, p. 3.

22 J. Bichot, Les politiques sociales en France au XX-e siècle, Paris, Armand Collin, 1997, p. 13. 
portés par une puissance collective, voire politique, leur permettant de faire valoir leurs droits. Selon une expression juste d'Aleksiei Petrovich Keppen, « les travailleurs, groupés en nombre significatif dans la même localité, se sont vite rendus compte qu'ils étaient une force reconnue ${ }^{23}$.

Aux XVIII et $X I X^{e}$ siècles, il existait en sciences sociales deux approches opposées pour résoudre le problème de la pauvreté : le darwinisme social et l'égalitarisme.

Le darwinisme social partait du postulat selon lequel «celui qui est faible est pauvre ${ }^{24}$. Les représentants de cette doctrine (Adam Smith, David Ricardo) considéraient la pauvreté comme une conséquence inévitable de la croissance démographique et du développement industriel. Certains d'entre eux (Herbert Spencer, Pierre-Joseph Proudhon), ayant appliqué les lois de la sélection naturelle et de l'évolution biologique à la société humaine, croyaient que la pauvreté était le moteur du développement de la personnalité.

Un autre représentant du darwinisme social, Thomas Robert Malthus, estimait pour sa part que " l'élimination de la pauvreté, des vices et autres catastrophes parmi la population, ne peut être réalisée qu'en réduisant davantage la croissance démographique, et non par des réformes politiques ou par un système d'égalité universelle, car ces réformes et ces systèmes provoquent une augmentation excessive de la population, c'est-à-dire la cause profonde des catastrophes $»^{25}$.

La seconde approche pour résoudre le problème de la pauvreté, fondée sur les idées de l'égalitarisme, a été présentée dans les travaux des Marxistes; Karl Marx naturellement, mais aussi Friedrich Engels, Vladimir Lénine, Gueorgui Plekhanov, etc.

Ainsi, Marx estimait que l'accumulation du capital entraîne une progression du prolétariat et que le destin de la majorité de la population restait la pauvreté. Dans le Chapitre XXV, «La loi générale de l'accumulation capitaliste » de son ouvrage Le Capital, il affirmait ainsi qu'il existe une interdépendance entre la richesse et la pauvreté : plus grande est l'accumulation de richesse par une classe de la société (parmi les propriétaires des moyens de production), plus l'accumulation de pauvreté dans la classe opposée est importante (dont le prolétariat) ${ }^{26}$. Selon Marx, cette contradiction pouvait être dépassée par l'élimination d'une société divisée en classes et par l'établissement du socialisme. Dans son ouvrage Critique $d u$ programme Gotha, Marx soulignait en outre que la société socialiste devrait allouer aux personnes inaptes au travail un certain nombre de moyens (comme une part du revenu total $)^{27}$.

L'idée de solidarité sociale, considérée comme étant la base originelle de l'assurance retraite, permet de dépasser les extrêmes des différentes approches évoquées ci-dessus. Elle implique une certaine part d'autonomie et de responsabilité

23 A.P. Keppen, Législation sociale de la France et de la Belgique, Saint-Pétersbourg, 1900, p. 3.

24 G.B. Chelnokova, Législation sociale visant à protéger contre la pauvreté en Russie: un guide scientifique et pratique, Moscou, 2009, p. 7.

25 T.-R. Malthus, L'expérience de la loi de la population, Moscou, 1895, p. 14.

26 K. Marx, Le capital, Moscou, 1955, Tome 1, p. 650.

27 K. Marx et F. Engels, Euvres, Moscou, 1986, Tome 19, p. 17. 
du salarié et, en même temps, suppose le partage des risques entre la communauté des travailleurs, ainsi que la participation des employeurs et de l'Etat dans le financement de l'assurance sociale.

L'économiste allemand Adolph Wagner, considéré comme l'un des fondateurs de la doctrine de l'assurance sociale, a énoncé plusieurs dispositifs théoriques, utilisés en Allemagne lors de l'élaboration des lois sur l'assurance sociale en 18821890, telles que la nécessité d'obliger légalement les employeurs à cotiser pour assurer leurs salariés contre les risques sociaux les plus courants - la maladie, les accidents du travail et la vieillesse ${ }^{28}$.

Développée aux $\mathrm{XIX}^{\mathrm{e}}$ et $\mathrm{XX} \mathrm{X}^{\mathrm{e}}$ siècles par des chercheurs et politiciens français, comme Auguste Comte, Emile Durkheim, Léon Duguit ou Léon Bourgeois, la doctrine de la solidarité (solidarisme) a eu un impact significatif sur la formation des institutions d'assurance sociale.

En particulier, Auguste Comte est considéré comme l'auteur de la doctrine des étapes du progrès social et l'un des fondateurs de l'idée de solidarité dans l'interaction sociale. Formulée dans son ouvrage Le système de la politique positive, ce concept a ensuite été utilisé par la science juridique. Comte estimait que le droit joue un rôle particulier dans la dissuasion ou dans la prévention des conflits sociaux, le but du droit étant de restreindre et de prévenir les dissensions entre les idées, les sentiments ou les intérêts qui existent dans la société. L'évolution incontrôlée de ces désaccords conduit, selon lui, à un ralentissement du progrès social.

Dans son ouvrage Sur la division du travail social, Durkheim a souligné que la division du travail entraîne l'augmentation de la dépendance de chaque citoyen à l'égard de la société, dans la mesure où les individus remplissent des fonctions complémentaires en matière de production sociale. Durkheim qualifiait de solidarité « organique » cette interconnexion entre membres de la société qui, contrairement à la solidarité mécanique de la société primitive, naît de la différenciation des individus et de la séparation de leurs fonctions dans la production sociale ${ }^{29}$.

S'appuyant sur le concept de solidarité, Duguit a développé la notion de « droit social ". C'est dans ses ouvrages que la doctrine de la solidarité s'est transformée en théorie politique et en construction juridique ${ }^{30}$. Selon lui, l'émergence d'une solidarité organique liée à la généralisation de la division du travail dans la société nécessite l'apparition d'un nouveau droit - le droit social - et l'exercice par l'Etat d'un nouveau type d'autorité induit par les exigences de solidarité (interdépendance) ${ }^{31}$. Le droit social devrait être fondé sur le fait que tous les membres de la société, en tant que parties de son ensemble, sont étroitement liés, dotés de droits et d'obligations mutuels. Dès lors, le droit social prévoit une assistance aux personnes dans le besoin. Refusant le principe contractuel de l'égalité, le droit social se fonde sur une telle compréhension de la justice, qui exige de mettre en œuvre des éléments

28 A. Wagner, La question sociale, Saint-Pétersbourg, 1906, p. 9.

29 E. Durkheim, De la division du travail social, Odessa, 1900, p. 55.

30 M. Borgetto et R. Lafore, La République sociale, Paris, 2000, p. 151.

31 L. Duguit, Droit social, droit individuel et transformation de l'État, Saint-Pétersbourg, 1909, p. 19. 
d'inégalité pour protéger les personnes en situation de dépendance, et soutenir de ce fait les individus "économiquement faibles »"

Selon Georges Gurvitch, le droit social est un droit consacré dans les formes supérieures d'interaction humaine, un droit qui favorise l'intégration dans les relations entre individus. L'incarnation du droit social est la législation sociale ${ }^{33}$. Enfin, Léon Bourgeois, Premier ministre français en 1895-1896, a élaboré un programme politique basé sur le principe de solidarité, qui impliquait une solidarité non seulement entre les individus, mais également entre les générations. Partant du concept d'interdépendance des individus, il conclut, dans son ouvrage Solidarité, qu'il existe un devoir de chaque citoyen envers la société, devoir qui peut être décrit dans le cadre du « contrat entre les générations $»^{34}$.

\section{II - LA CONSÉCRATION DE DIFFÉRENTS CONCEPTS DANS LA LÉGISLATION SUR LES RETRAITES}

Les premières lois relatives à l'assurance retraite obligatoire, adoptées dans les pays européens à la fin du $X I X^{e}$ et au début du $X X^{e}$ siècle, ont tenté de mettre en œuvre certains des concepts évoqués ci-dessus. II convient de noter le rôle particulier de la législation allemande sur les assurances, élaborée avec la participation du Chancelier Bismarck dans les années 1880. L'expérience allemande dans ce domaine est devenue " un modèle contagieux $»^{35}$.

En 1889, le Parlement allemand a adopté une loi sur l'assurance des travailleurs contre les incapacités et la vieillesse ${ }^{36}$. Cette loi prévoyait l'assurance obligatoire des travailleurs dont le revenu annuel ne dépassait pas 2.000 Deutsche Mark. Après 5 années de paiement des cotisations, ce type d'assurance donnait droit à une pension d'invalidité et de vieillesse dès 70 ans. Les fonds d'assurance étaient constitués par des contributions paritaires provenant pour chaque tiers des employeurs, des travailleurs et de l'État.

S'intéressant à la fonctionnalité de cette loi, un homme politique russe du XIXe siècle, Alexandre Goldenweiser, a considéré que les innovations les plus importantes y étaient recensées : I'introduction du principe de l'assurance obligatoire pour protéger les salariés, l'obligation de verser des contributions aux fonds d'assurance pour une classe sociale qui ne les utilisera pas (les employeurs), la participation de l'Etat au financement de l'assurance sociale et à l'organisation de ses institutions ${ }^{37}$.

32 M. Borgetto et R. Lafore, op.cit., p. 137.

33 G. Gurvitch, L'idée du droit social, Paris, 1932, p. 119.

34 L. Bourgeois : http://www.senat.fr/senateur-3emerepublique/bourgeois leon0647r3. html\#1889-1940

35 V. D. Roik, Assurance sociale: économie, finances et gestion, Moscou, 2014, p. 9.

36 "Loi sur l'assurance invalidité et vieillesse », in Gazette Allemande du droit du Reich, 1889, $n^{\circ} 13$, Sections 97-144.

37 A. S. Goldenweiser, Législation sociale de l'Empire allemand: assurance des travailleurs contre la maladie, les accidents, en cas de perte de capacité de travail et de vieillesse, Moscou, 2016. p. 4. 
Dans les années 1870-1880, les députés du Parlement français travaillaient sur l'élaboration d'une loi concernant l'assurance retraite. Le premier projet, préparé en 1879, posait le principe d'une cotisation salariale obligatoire, doublée d'une cotisation patronale identique et augmentée d'une contribution de l'Etat. L'âge de départ à la retraite était précoce, car « à 55 ans, l'ouvrier doit être considéré comme ne pouvant plus rien produire $»^{38}$.

D'autres propositions ont été présentées en 1881, 1882 et 1886. Cependant, l'idée d'introduire en France un système d'assurance sociale obligatoire s'est heurtée à une sérieuse opposition. Perçue comme une tentative d'assujettir l'indépendance personnelle et la liberté individuelle des citoyens, elle ne s'est finalement concrétisée que grâce aux efforts d'une lutte révolutionnaire contre l'ancien régime ${ }^{39}$. Les travaux sur le projet de loi français relatif aux retraites ont duré environ 20 ans. Adoptée en 1910, la loi sur les retraites ouvrières et paysannes ${ }^{40}$ instituait un régime de retraite obligatoire pour les salariés gagnant moins de 3.000 Francs par an. La cotisation était paritaire, un salarié et un employeur payaient la même somme : 9 Francs par an pour les hommes; 6 Francs pour les femmes ; 4,50 francs pour les travailleurs de moins de 18 ans. L'âge de départ à la retraite était fixé à 65 ans, puis abaissé à 60 ans en 1912.

Selon Jacques Bichot, " la longueur de la gestation ne garantit nullement la qualité du travail de conception et de préparation. Si la loi de 1910 ne fut que peu appliquée, c'est parce que le travail législatif dont elle était le fruit a manqué de sérieux ${ }^{41}$. En effet, les travailleurs se soustrayaient à leur obligation de cotiser, l'inflation contribuait à la dépréciation des moyens accumulés et, de plus, les syndicats (y compris la Confédération générale du travail) se sont opposés à l'attribution d'une partie des cotisations aux travailleurs. Une nouvelle tentative de mise en place d'une assurance retraite obligatoire en France fut menée avec la loi de 1928. Néanmoins, ce texte a rencontré une résistance active de la part des employeurs et de certaines catégories de travailleurs (en particulier les professions médicales), car il traitait non seulement des assurances vieillesse mais également de l'assurance maladie. La loi ne fut donc jamais appliquée.

Il fallut attendre la promulgation de la loi de 1930 instaurant les assurances sociales pour les salariés de l'industrie et du commerce, pour que l'assurance retraite au niveau national soit introduite. Cette loi prévoyait la possibilité d'une assurance en cas de vieillesse, maladie, maternité, invalidité ou décès. Le régime obligatoire était prévu pour les travailleurs dont le salaire ne dépassait pas 15.000 Francs par an. Le système d'assurance était basé sur le principe de la capitalisation. En outre, la loi de 1930 prévoyait une possibilité de réduction des cotisations d'assurance sociale compensées par l'État; elle a également augmenté de manière significative le " plafond " des salaires pour le paiement des cotisations, amplifiant de ce fait le

38 J. Bichot, op.cit., p. 26.

39 H. Adan, La question des assurances contre les accidents devant la Commission du travail, Bruxelles, 1888, p. 31.

40 Loi du 5 avril 1910 sur les retraites ouvrières et paysannes [annotée et commentée par M. A. Goineau], Paris, 1910.

41 J. Bichot, op.cit., p. 26. 
nombre d'assurés. C'est pourquoi cette loi fut soutenue, tant par le Parlement que par la société.

La loi britannique sur les pensions de $1908^{42}$ représente un autre modèle de protection sociale des personnes âgées. Promulguée - comme en France - à la suite d'une longue lutte politique, cette loi établit un système de pensions de vieillesse financé par l'État.

En Grande-Bretagne, les premières propositions pour l'introduction de la pension universelle sont apparues dans les années 1870. Les discussions tournaient alors autour de deux projets de réforme des retraites, un projet d'assurance et un projet d'assistance, dont les principales différences concernaient la proportion de la part contributive et de la non-contributive (contributory ou non-contributory pension), le caractère obligatoire ou volontaire de la cotisation, ou encore la couverture des retraités (avec des restrictions sur le revenu, l'âge ou le comportement moral).

Ainsi en 1878, William Lewery Blackley ${ }^{43}$ a proposé un projet d'assurance retraite selon lequel des cotisations obligatoires aux fonds de retraite devaient être prélevées sur les salaires de tous les jeunes travailleurs de moins de 20 ans. Selon ses calculs, le travailleur pouvait transférer la somme de 15 livres sterling en une année, ce qui était suffisant pour lui octroyer une allocation de 8 livres par semaine en cas de maladie, et une retraite de 4 livres après 70 ans. En revanche, aucune participation de l'État n'était prévue ${ }^{44}$.

Ce projet a été rejeté, comme plusieurs autres, principalement critiqués au regard du fait que le montant élevé des cotisations ne permettait pas aux travailleurs à bas salaire de participer au système d'assurance; que les femmes étaient exclues du système et que les personnes âgées dans le besoin ne pourraient pas bénéficier des prestations pendant les premières années de la réforme (une durée minimale de cotisation était nécessaire). Par conséquent, une loi a été adoptée en Grande-Bretagne pour instituer le principe du financement public (budgétaire) des pensions via l'introduction de nouveaux impôts. Cette loi prévoyait des conditions qui réduisaient considérablement le cercle des bénéficiaires des pensions : un âge de départ à la retraite élevé (70 ans), le principe de nécessité, le « test du comportement moral » (character test).

Puis en 1925, la Grande-Bretagne a introduit une assurance vieillesse pour les salariés ayant atteint l'âge de 65 ans et dont le revenu annuel ne dépassait pas 250 livres sterling. Les employeurs, les salariés et l'Etat devaient participer au financement de ce système. C'est ainsi que, vers le milieu du $X X^{\top}$ siècle, un régime de retraite basé sur les principes de l'assurance a été mis en place au Royaume-Uni.

S'agissant de la Russie, le processus de formation d'un système d'assurance sociale a débuté au XXe siècle. En 1901, les Règles temporaires sur les pensions des travailleurs des mines furent adoptées. Puis le 23 juin 1912, une série de lois

42 "Old-Age Pensions Act 1908 », in Later Nineteenth Century: 1868-1919, Londres, 1969, p. 208.

43 W. L. Blackley, Clerc de l'Église d'Angleterre et réformateur social, 1830-1902.

44 Ch. Booth, Old Age Pensions and the Aged Poor. A Proposal by Charles Booth, Londres, 1899 , p. 30. 
est promulguée ${ }^{45}$, permettant d'établir les bases de l'assurance sociale en cas de maladie et d'accident du travail. En revanche, l'assurance retraite n'était pas prévue par ces lois et leur champ d'application restait très limité.

Le Parti ouvrier social-démocrate de Russie (POSDR) a vivement critiqué ce texte au moment de sa discussion au Parlement.

Dès janvier 1912, lors de sa $6^{\text {e }}$ Conférence à Prague, le POSDR s'est emparé de tous les points faibles du texte et a adopté son propre programme d'assurance, basé sur les principes suivants:

- l'assurance sociale devrait, en toutes circonstances, couvrir les travailleurs de leur perte de capacité de travail (accident du travail, maladie, vieillesse, invalidité, etc.);

- tous les assurés devraient recevoir une prestation basée sur le principe d'une indemnisation totale en fonction du salaire ;

- tous les frais d'assurance devaient incomber aux entrepreneurs et à l'État (le salarié ne devant pas cotiser);

- tous les types d'assurance devaient être gérés par des organismes d'assurance unifiés ${ }^{46}$.

Ce programme qui incarnait les idées marxistes reçut, après la révolution de 1917, sa consécration législative.

En Russie soviétique, l'assurance sociale a perdu son caractère assurantiel et a commencé à être financée par le budget de l'État, en dépit du fait que la dénomination d'assurance "sociale " ait été conservée. Dans les années 1920-1930, un certain nombre de décrets gouvernementaux furent adoptés, prévoyant le droit à une pension de vieillesse pour certaines catégories de travailleurs (mines, métallurgie, transports ferroviaires et fluviaux, etc.). Puis, ces normes ont été étendues à d'autres catégories de salariés et, le 11 février 1930, le Commissariat du Travail de I'URSS a approuvé les Règles de la sécurité sociale pour la vieillesse ${ }^{47}$ fixant l'âge de la retraite pour la plupart des catégories de travailleurs à 60 ans pour les hommes et 55 ans pour les femmes (respectivement 25 et 20 ans d'ancienneté).

Pour conclure, la législation sur les retraites adoptée durant la première moitié du XXe siècle en France, en Grande-Bretagne et en Russie, résulte d'une lutte idéologique et politique. Les lois françaises sur les pensions de 1910, 1928 et 1930, ont ainsi fortement été influencées par la législation de Bismarck et ont contribué à la genèse du système moderne d'assurance vieillesse. La loi britannique de 1908 sur les retraites a, pour sa part, incarné le concept d'assistance en prévoyant des pensions minimales financées par l'État à un nombre limité de personnes âgées.

Dans la Russie pré-révolutionnaire, la loi sur l'assurance retraite n'a pas été adoptée, contrairement aux lois sur l'assurance maladie et sur l'assurance invalidité temporaire. Puis, la législation promulguée en Russie soviétique durant la première

45 Lois du 23 juin 1912 "sur l'assurance des travailleurs contre les accidents du travail ", "sur la prestation des travailleurs en cas de maladie », etc.

46 Lénine, Oeuvres complètes, Moscou, 1985, Tome 21, p. 146.

47 Bulletin du Commissariat du Travail de I'URSS, 1930, n 10, p. 234. 


\section{LA LÉGISLATION SUR LES RETRAITES EN RUSSIE}

moitié du XXe siècle a reflété le concept égalitaire (marxiste) qui prévoyait la sécurité sociale de tous les travailleurs en situation de vieillesse, financée par le budget de l'État.

Plus tard, le Rapport Beveridge ${ }^{48}$ en 1942 aura un impact significatif sur le développement des systèmes de retraite. Les principes-clés formulés par Beveridge - universalité et uniformité - formeront ainsi la base du système de retraite britannique et influenceront également les réformes sociales engagées en France (1944-1958).

Enfin, différents actes internationaux vont significativement impacter l'élaboration de la législation sur les retraites, comme la Charte de l'Atlantique de 1941, la Déclaration de Philadelphie de l'Organisation internationale du Travail de 1944, la Convention $n^{\circ} 102$ concernant la sécurité sociale.

48 W. H. Beveridge, économiste et homme politique britannique, 1879-1963.

\section{ANNA ALEKSANDROVA}

PhD, chargée de cours à l'Institut de droit de l'Université d'Etat de Penza, Russie.

Thèmes de recherche : Droit comparé constitutionnel et droit comparé social.

\section{Publications:}

A. Aleksandrova, "Actualités juridiques internationales. Fédération de Russie », Revue de droit comparé du travail et de la sécurité sociale, 2019/3, p. 196.

$\sim$ A. Aleksandrova, " Le droit social dans une société post-industrielle ", Revue de droit comparé du travail et de la sécurité sociale, 2018/1, p. 92. 